\title{
LX. On the law of force between electric currents
}

\section{H.W. Watson \& S.H. Burbury}

To cite this article: H.W. Watson \& S.H. Burbury (1881) LX. On the law of force between electric currents, Philosophical Magazine Series 5, 11:70, 451-466, DOI: 10.1080/14786448108627042

To link to this article: http://dx.doi.org/10.1080/14786448108627042

$$
\text { 册 Published online: } 28 \text { Apr } 2009 .
$$

Submit your article to this journal

Џ Article views: 2

Q View related articles ¿ 
$A$ detailed account of the results obtained by this method, including determinations of the electric distribution on metallic conductors, will be found in a paper recently communicated by me to the Royal Society.

Dulwich College, April 1881.

LX. On the Law of Force between Electric urrents. By H. W. Watson and S. H. BurburY*.

1. TTHE laws of mutual action between electric currents, or

1 between separate elements of electric currents, have been investigated by Ampère, and, following him, by F. E. Neumann, Weber, Helmholtz, Clausius, and others. Their object has been to discover a law of force between the elements which should give results in accordance with facts established, or supposed to have been established, by experiment. Ampere's experimental data are given by Professor Tait in his work on Quaternions, second edition, p. 250, as follows, using his own words :-

"I. Equal and opposite currents in the same conductor produce equal and opposite effects on other conductors; whence it follows that an element of one current has no effect on an element of another which lies in the plane bisecting the former at right angles.

"II. The effect of a conductor bent or twisted in any manner is equivalent to that of a straight one, provided that the two are traversed by equal currents, and the former nearly coincides with the latter.

"III. No closed circuit can set in motion an element of a circular conductor about an axis through the centre of the circle and perpendicular to its plane.

"IV. In similar systems traversed by equal currents the forces are equal."

To these canons, deduced from experiments of Ampère, may now be added:-

V. Oersted's experiments, showing that the action of a closed circuit is equivalent to that of a magnetic shell of proper strength whose boundary coincides with that of the circuit.

Also VI., an experiment of which the details are given in Maxwell's 'Electricity,' vol. ii. p. 149, which is supposed to prove directly that the force exerted by any closed electric circuit on any element of another circuit is always normal to the element. The law of force, whatever it be, must satisfy these conditions.

* Communicated by the Authors. 
2. On these canons we make the following observations.

With regard to I., it will be observed that it consists, first, of a statement of fact-mamely, that a reversal of one current reverses the effect; secondly, of an inference supposed to follow from the observed fact-namely, that an element of one current has no effect on an element of another which lies in the plane bisecting the former at right angles. Let the first eloment be at the origin in direction $x$, and the second anywhere in the plane of $y, z$. If, when the first element is in the positive direction, the second is attracted, it follows, by the general principle enunciated, that were the first element in the negative direction, the second would be repelled. Now we cannot imagine any reason why in the former case the force should be an attraction and in the latter a repulsion, any more than the converse. Hence it is concluded, no doubt rightly, that there can be no force tending to move the second element in the plane of $y, z$.

But it should here be observed that the reasoning would not apply to a couple tending to turn the second element round an axis without changing the position of its centre. The hypothesis, for instance, that the first element tends to turn the second into a position parallel to the first, agrees with the general law enunciated in I., and is not open to à priori objection.

II. is equivalent to the principle, which is universally assumed in all treatises on the subject, that any elementary current may be replaced by its components, the middle points of the components being identical with that of the element.

IV. leads to the conclusion, as shown in Maxwell's 'Electricity,' vol. ii., that the forces of attraction between two elements are inversely proportional to the square of the distance between them.

V. It follows from Oersted's experiments, that the mutual action of two closed electric circuits is the same as that of two magnetic shells bounded by the circuits. It must therefore have a potential; and such potential must be of the form

$$
\mu i i^{\prime} \iint \frac{\cos \epsilon}{r} d s d s^{*}
$$

in which $i, i^{\prime}$ are the strengths of the currents, and $\epsilon$ is the angle, and $r$ the distance between an element $d s$ of the one,

* We take the positive sign in the same way as it is usual to say the potential of unit mass of matter at distance $r$ is $\frac{1}{r}$. 
and an element $d s^{\prime}$ of the other circuit, and $\mu$ is a constant depending on the nature of the medium in which the shells are placed.

3. If we were here to assume that the potential of mutual action between each pair of elementary currents is of the same form,

$$
\mathrm{V}=\mu i i^{\prime} \frac{\cos \epsilon}{r} d s d s^{\prime},
$$

we should of course obtain the correct value for the potential of two closed circuits; and therefore this assumption would satisfy all the results obtained from experiments with closed circuits. This form of mutual potential was proposed by F. E. Neumann, but seems to have been abandoned, because it would not satisfy the results obtained, or supposed to have been obtained, in experiments with open currents. Now, according to the views of Maxwell, no such thing can exist within the range of our experiments as an unclosed current, because the current, if not closed by conductors, closes itself by means of change of displacement in the dielectric. If this be true, no experiments can ever lead to results inconsistent with the above simple law of potential. Further, even without assuming the truth of Maxwell's theory, it appears to us that the experiments which were supposed to be inconsistent with the above law admit of interpretation consistent with it, as we hope to show.

4. It was believed, however, to be established by experiment, that the attractive force exerted by any closed circuit upon any element of another current is always normal to the element (canons III. and VI.). According to the above law of F. E. Neumann, the impressed force exerted by the closed circuit on an isolated element would not necessarily be normal to it. It was thought necessary, therefore, to invent a law of force between two elementary currents which should satisfy this supposed experimental result, and at the same time should give the correct value for the potential of two closed circuits.

According to F. E. Neumann's law, the force between two elements of given strength depends only on their distance and the angle, $\epsilon$, which their directions make with one another; it is independent of the angles $\theta$ and $\theta^{\prime}$ which their directions make with $r$, the line joining them. But, by a known geometrical theorem,

$$
\iint \frac{\cos \epsilon}{r} d s d s^{\prime}=\iint \frac{\cos \theta \cos \theta^{\prime}}{r} d s d s^{\prime}
$$

for any two closed curves in space. Hence, if we assume for 
the potential of two elementary currents $i d s$ and $i^{\prime} d s^{\prime}$ the form

$$
i^{\prime}\left\{\mathrm{A} \frac{\cos \epsilon}{r}+\mathrm{B} \frac{\cos \theta \cos \theta^{\prime}}{r}\right\} d s d s^{\prime},
$$

where A and B are any constants, we should for any pair of closed circuits obtain as the potential an expression proportional to

$$
i i^{\prime} \iint \frac{\cos \epsilon}{r} d s d s^{\prime},-
$$

that is, practically identical with that obtained from F. E. Neumann's law. But the force exerted by a closed circuit on a separate element of another would not be identical in the two cases ; and it may by proper choice of the constants A and $B$ be made always normal to the element.

5. Ampere's solution of the problem is as follows, assuming a force and not a potential between two elementary currents. Let $i \cos \theta d s, i^{\prime} \cos \theta^{\prime} d s^{\prime}$, according to the usual notation, be the component parts of the elements resolved in $r$, the line joining their centres ; and $i \sin \theta d s, i^{\prime} \sin \theta^{\prime} \cos \phi d s^{\prime}$, the component parts perpendicular to $r$ in the plane'of $r$ and $d s$. Then it is assumed that the two radial components attract each other with a force in the direction of $r$ varying inversely as the square of the distance, viz.

$$
\frac{a}{r^{2}} \cos \theta \cos \theta^{\prime} i i^{\prime} d s d s^{\prime},
$$

and the two transverse components attract each other with a force in the direction of $r$, viz.

$$
\frac{b}{r^{2}} \sin \theta \sin \theta^{\prime} \cos \phi i i^{\prime} d s d s^{\prime},
$$

where $a$ and $b$ are constants. It is then shown that if the relation between $a$ and $b$ be $2 a+b=0$, the desired result will follow -namely, that the force exerted by any closed circuit on any element of a current is always normal to the element. This relation, then, satisfies Professor Tait's canon III. above given, and also satisfies VI. It will be found also to lead to the correct expression for the mutual potential of two closed circuits, and therefore satisfies all experimental conditions.

Ampère's results may be concisely expressed as follows; viz. the action of $i d s$ upon $i^{\prime} d s^{\prime}$ is a force in the direction of the line, $r$, which joins them, and whose intensity is

$$
\frac{\ddot{i i^{\prime}}}{\sqrt{\boldsymbol{r}}} \cdot \frac{d^{2} \sqrt{r}}{d s d s^{\prime}} d s d s^{\prime}
$$


This law of force gives no potential of the action of one element upon another; but, as we have said, it gives the potential

$$
i i^{\prime} \int \frac{\cos \epsilon}{r} d s d s^{\prime}
$$

for the mutual action of two CLOSED circuits.

6 . Weber has further shown that if an electric current be supposed to consist of positive electricity moving with velocity $v$ in one direction, and an equal quantity of negative electricity moving with the same velocity in the opposite direction, then a certain hypothesis regarding the force exerted on one another by particles of electricity in motion not only leads to Ampère's law of force, but also explains the ordinary phenomena of induction by variation of the primary current, or by variation of the position of the circuits. Weber's hypothesis is that the mutual potential of two particles of electricity $e$ and $e^{\prime}$ is

$$
\frac{e e^{\prime}}{r}\left\{1-\frac{1}{2 c^{2}}\left(\frac{d r}{d t}\right)^{2}\right\}
$$

where $c$ is a constant. If the particles be at rest, $\frac{d r}{d t}=0$, and this expression gives the ordinary electrostatical potential $\frac{e e^{\prime}}{r}$. If they be in motion, it will be found to lead to Ampère's law, as shown by Briot*. The coincidence appears at first sight remarkable, and has done much to facilitate the acceptance of Ampère's results as well as Weber's. It will be seen, however, on further considering the subject, that Ampère and Weber both start from the same fundamental assumption with regard to the nature of the action between two elementary currents-viz. that it depends not only on their directions relative to each other, but also on their directions relative to the line joining them. This may perhaps account for their leading to the same result.

7. Stefan has shown $\dagger$ that, assuming the only forces acting to be, as Ampère assumes,

and

$$
\frac{a}{r^{2}} \cos \theta \cos \theta^{\prime} i i^{\prime} d s d s^{\prime}
$$

$$
\frac{b}{r^{2}} \sin \theta \sin \theta^{\prime} \cos \phi i i^{\prime} d s d s^{\prime},
$$

then, if there be a potential for two closed circuits, such poten-

* Theorie mécanique de la Chaleur, chap. ix.

$\dagger$ Sitzungsberichte, Vienna, 1869. 
tial can have no other form than

$$
\mathrm{V}=\mu i i^{\prime} \iint \frac{\cos \epsilon}{r} d s d s^{\prime}
$$

and, further, that there can be no potential at all unless the relation between the constants $a$ and $b$ be $2 a+b=0$, which was the relation obtained by Ampère from different reasoning. It thus appears again that Ampère's law leads to the correct expression for the potential.

Stefan has, further, considered the consequences of assuming mutual action in the nature of attraction or repulsion between the radial component of one, and the transverse component of the other element. The radial component of $d s$ is $i \cos \theta d s$; the transverse component of $d s^{\prime}$ in plane of $r$ and $d s$ is $i^{\prime} \sin \theta \cos \phi d s^{\prime}$. If we suppose $i \cos \theta d s$ to exert on $i \sin \theta \cos \phi d s^{\prime}$ a force tending to move it in the direction of $r$,

$$
i i^{\prime} \frac{c}{r^{2}} \cos \theta^{\prime} \sin \theta^{\prime} \cos \phi d s d s^{\prime},
$$

and, in like manner, the transverse component of $d s$, namely $i \sin \theta d s$, to exert on $i^{\prime} \cos \theta^{\prime} d s^{\prime}$, the radial component of $d s^{\prime}$, a force in direction of $r$,

$$
i i^{\prime} \frac{d}{r^{2}} \sin \theta \cos \theta^{\prime} d s d s^{\prime},
$$

where $c$ and $d$ lare two new constants, then Stefan shows that the potential, if there be a potential, must have the same form as before, and that there can be no potential unless

$$
2 a+b+c-2 d=0 \text {, }
$$

which, by making $c=0$ and $d=0$, includes Ampère's law as a particular case.

8. Carl Neumann, in a very elaborate memoir*, has deduced the following as the attractive force between the two elementary currents $i d s, i^{\prime} d s^{\prime}$, viz.

$$
-i i^{\prime}\left\{\frac{2}{r^{2}} \cos \epsilon-\frac{1}{r^{2}} \cos \theta \cos \theta^{\prime}\right\} d s d s^{\prime},
$$

and this withont assuming that the closed circuit exerts a normal force on each element of another current.

C. Neumann, however, excludes from his consideration " couple-action," whereby one element may tend to turn another round an axis without altering its position in space.

9. We may here add that Clausius considers that the $x$

* Ueber die den Kräften electrodynamischen Ursprungs zuzuschreibenden Elementargesetze: Leipzig, 1873. 
component of the force which a moving particle of electricity $e$ exerts on another, $e^{\prime}$, is expressed by the formula

$$
e e^{\prime}\left\{\frac{d \frac{1}{r}}{d x}\left(-1+k\left(\frac{d x}{d t} \frac{d x^{\prime}}{d t}+\frac{d y}{d t} \frac{d y^{\prime}}{d t}+\frac{d z}{d t} \frac{d z^{\prime}}{d t}\right)\right)-k \frac{d}{d t}\left(\frac{1}{r} \frac{d x}{d t}\right)\right\}
$$

where $x, y, z$ relate to the first and $x^{\prime}, y^{\prime}, z^{\prime}$ to the second particle (see Phil. Mag. October 1880).

10. It seems that the Continental physicists consider the force between two elementary currents as a force of translation only, excluding from their consideration "couple-action," whereby one element tends to turn the other round an axis without altering its position in space. But such couple-action results immediately, as will be shown later, from F. E. Neumann's law of potential.

11. Maxwell has treated the subject in a different way. While giving Ampère's reasoning and his results, as well as those of Weber, he founds his own theory on the hypothesis that in nature all circuits are closed circuits, and proceeds to obtain the known results of experiment, by the application of ordinary dynamical methods to the theory of closed circuitsthat is, that if the circuits be not closed by conductors, there arise changes of displacement of electricity in dielectrics which have the effect of closing the currents.

12. Now, if it be true, as Maxwell teaches, that in nature, or within the range of our experiments, all currents are closed, there can be no necessity for devising elaborate laws of force. We have only to assume for each pair of elements the potential

$$
\nabla=\mu i i^{\prime} \frac{\cos \epsilon}{r} d s d s^{\prime},
$$

and we cannot fail to obtain results in accordance with experiment, and, in fact, exactly the same results as are obtained from any of the more complicated laws above stated; so that, on this view, no experiment could furnish any reason for accepting one law rather than the other. It appears to us, then, that if Maxwell's theory, that all currents are closed, be accepted, the law that, for each pair of elementary currents,

$$
\mathrm{V}=\mu i i^{\prime} \frac{\cos \epsilon}{\gamma^{\prime}} d s d s^{\prime}
$$

ought to find provisional acceptance also.

13. But as Maxwell's theory is not accepted universally, we proceed to examine some of the experimental evidence on which the doctrine is based, that a closed circuit exerts a 
normal force only on every elementary current (canons III. and VI.), this being the only experimental fact inconsistent with the simple law.

The experiments quoted in support of this are the following:-

(1) A current in a circular conductor, movable about an axis through its centre perpendicular to its plane, remains unmoved in the field of another closed circuit.

(2) A current in a rectangular conductor A BCD, movable about one side $A D$, which coincides with a line through the centre, $O$, of a circular conductor, the line $\mathrm{AD}$ being perpendicular to the plane of the latter, remains immovable when a current passes through the circular conductor.

(3) (Maxwell, vol. ii. p. 149, cited above.) A circular conductor, C D, movable about its centre in its own plane, passes through two mercury-cups fixed at A and $B$, at which a current enters and leaves the conductor respectively; and no motion ensues when a closed current is brought into the neighbourhood.

Now, in point of fact, all these three experiments would be equally satisfied by any law which gave a potential between two currents. For in (1) and (2) no possible displacement of the movable circuit could alter that potential, if it existed, as is obvious from the symmetry of the arrangements in the original and displaced positions respectively. Since, therefore, no displacement could alter the potentials if they existed, it follows that if there be a potential there would be no force producing motion.

The same thing is true of No. 3, although at first sight not so evident; for since the current enters and leaves the conductor at the points $A$ and $B$ fixed in space, and the arc A B is fixed in space, it follows that, notwithstanding any motion of the conductor, the current remains fixed in space, and the potential must remain the same whether the conductor be moved or not. Hence there can be no tendency to move the conductor.

These three experiments, therefore, do not prove that the 
law of action between current-elements is necessarily such that the resultant action of a closed circuit on an element is normal to that element; they would be equally satisfied by any law of action giving a potential between two closed circuits.

14. Indeed it may be shown directly that, whether there be any tangential action or no, it never can be manifested by experimenting upon a closed circuit; and therefore, if all circuits be necessarily closed circuits, it follows that such tangential action never can be manifested.

For such closed circuit must be infinitely flexible and infinitely extensible (at least in the neighbourhood of the element acted on), otherwise the element will be influenced by the mechanical action of the adjacent parts of its own circuit; and if it be perfectly flexible and extensible, no virtual motion of any element in the direction of its length can alter the value of the potential

$$
\iint \frac{\cos \varepsilon}{r} d s d s^{\prime}
$$

15. Again, it is generally stated to be a deduction from Ampère's law, that the action of a solenoid of currents of innitely small section and indefinitely extended in one direction, upon an element of a current, is a force perpendicular to the plane passing through the element and the extremity of the solenoid, varying inversely as the square of the distance of the element from that extremity, and directly as the sine of the angle between the element and that distance. Ampère's law would give exactly this result, while that now proposed would not do so, for an element considered alone, $i$. e. otherwise than as part of a closed or infinite current. It is clear, for the reasons mentioned above, that no experiment can give a result in this form. What experiment really proves is, that in the case of a closed circuit or infinitely extended rectilinear current, the action on the solenoid is the same as if that of each element of the current followed the above law. And the law of F. E. Neumann leads exactly to the same conclusion. For let the infinite current be of strength $i$ in the axis of $z, z$; then it is evident by integration that Ampère's law of elementary current action would lead to a potential energy between the whole current and the solenoid of the form $i \tan ^{-1} \frac{y}{x}$, or, more generally, when the solenoid is not infinite, to the form

$$
i\left\{\tan ^{-1} \frac{y}{x}-\tan ^{-1} \frac{b}{a}\right\} \text {. }
$$

For according to the law now proposed, the potential between 
the cu rrent-element $i \delta z$ and one of the solenoid currents, whose strength is $i^{\prime}$, would be

$$
i i^{\prime} \delta z \int \frac{\cos \epsilon}{r} d s^{\prime}
$$

the integration being taken round the elementary circuit. By Stokes's theorem this becomes

$$
\frac{i i^{\prime} A(l y-m x)}{x^{3}}
$$

where $\mathrm{A}$ is the area of the solenoid-section, $l, m, n$ the direction-cosines of its normal, and $r$ the distance of its centre from $\delta z$. Therefore the potential energy between the whole infinite current and this section of the solenoid is $V$, where

$$
\begin{aligned}
\mathrm{V} & =\mathrm{A} i i^{\prime} \int_{-\infty}^{\infty} \frac{l y-m x}{\left(x^{2}+y^{2}+z^{2}\right)^{\frac{3}{2}}} d z \\
& =\mathrm{A} i i^{\prime} \frac{l y-m x^{*}}{x^{2}+y^{2}} .
\end{aligned}
$$

And the potential of the whole solenoid is

$$
\operatorname{Aii^{\prime }} \int \frac{y \frac{d x}{d s}-x \frac{d y}{d s}}{x^{2}+y^{2}} \cdot d z
$$

or

$$
\operatorname{Aii}^{\prime}\left\{\tan ^{-1} \frac{y}{x}-\tan ^{-1} \frac{b}{a}\right\} \text {. }
$$

16. Again, it is generally stated that the action of a particle of imaginary magnetic matter upon a current-element varies as the sine of the angle between the distance of the particle from the element and the element directly, and as the square of that distance inversely, and is perpendicular to the plane passing through the particle and the element. For the reasons mentioned in the case of the solenoid, it is obvious that no such law of action as this can be experimentally proved to exist between a current-element and a magnetic pole; but the equivalent law of action between an infinite current and a particle of imaginary magnetic matter may be deduced from the law of current-action now proposed, combined with the experimental fact that the potential of a current-circuit and a magnetic shell is equivalent to that between the circuit and the current bounding the shell of suitable intensity. For if $i$ be the intensity of a current in the axis of $z$, and $i^{\prime}$ that in the bounding circuit of a plane magnetic shell of very small area $A$, it will follow, as in the case last considered, that the poten- 
tial between the shell and current is

$$
\mathrm{A} i i^{\prime} \frac{l y-m x}{x^{2}+y^{2}}
$$

the symbols having the same meaning as before.

If, now, the potential of positive magnetic matter on one face of the area $\mathrm{A}$ relative to the infinite current be $\mu \mathrm{A} i \phi(x, y)$, that of the negative matter on the opposite face will be

$$
-\mu \mathrm{A} i \phi(x, y)-\mu \mathrm{A} i\left\{\frac{d \phi}{d x} l+\frac{d \phi}{d y} m\right\} h,
$$

where $h$ is the thickness of the shell; and the resulting potential is

$$
-\mu \mathrm{A} i\left\{\frac{d \phi}{d x} l+\frac{d \phi}{d y} m\right\} h
$$

But this has been shown to be equal to

$$
\mathrm{A} i i^{\prime} \frac{l y-m x}{x^{2}+y^{2}}
$$

Therefore these expressions must be equal for all values of $l$ and $m$;

and

$$
\begin{aligned}
& \therefore \mu h \frac{d \phi}{d x}=-\frac{i^{\prime} y}{x^{2}+y^{2}}, \quad \mu h \frac{d \phi}{d y}=+\frac{i x}{x^{2}+y^{2}} ; \\
& \therefore \phi(x, y)=\frac{i^{\prime}}{\mu h} \tan ^{-1} \frac{y}{x},
\end{aligned}
$$

$$
\mu \mathrm{A} i \phi(x, y)=\frac{\mathrm{A} i \ddot{i}^{\prime}}{h} \tan ^{-1} \frac{y}{x},
$$

giving the law required.

We have here shown in detail that these two results are deducible from the law of F. E. Neumann; but it is evident that we might have inferred this without further proof from the fact that we are throughout dealing with complete circuits, for which it has been already shown that the mutual potential is in all cases the same as that given by Ampère's law.

17. The adoption of the above law for the potential of two elementary currents, viz.

$$
\mathrm{V}=\mu i i^{\prime \prime} \frac{\cos \epsilon}{r} d s d s^{\prime}
$$

agrees perfectly with Maxwell's treatment of the subject, although Maxwell nowhere distinctly states the existence of the law, perhaps on account of its conflict with canon III. mentioned at the beginning of this paper, the validity of which

Plil. Mag. S. 5. Vol. 11. No. 70. June 1881. 2L L 
canon we have been calling in question. Moreover this law (if not disproved by some decisive experiment) would greatly simplify the mathematical treatment of electrodynamics.

Since for two elements

we have

$$
\mathrm{V}=\mu i i^{\prime} \frac{\cos \epsilon}{r^{\prime}} d s d s^{\prime}
$$

$$
\frac{d V}{d r}=-\mu i \ddot{i}^{\prime} \frac{\cos \epsilon}{r^{2}} d s d s^{\prime}
$$

For parallel currents in the same direction $\cos \epsilon=1$; in opposite directions, cos $\epsilon=-1$. Therefore, "Parallel elementary currents, if in the same direction, attract each other with a force varying inversely as the square of the distance; if in opposite directions, repel each other with the same force."

For perpendicular currents, $\cos \epsilon=0$; therefore $\frac{d \mathrm{~V}}{d r}=0$, or "Elementary currents at right angles to each other exert on each other no attractive or repulsiveiforce."

Again,

$$
\frac{d \mathrm{~V}}{d \epsilon}=-\mu i i \frac{\sin \epsilon}{r} d s d s^{\prime} .
$$

For parallel currents, $\sin \epsilon=0$; for perpendicular currents, $\sin \epsilon=1$. Therefore "Parallel elementary currents exert on each other no couple-action."

"Elementary currents at right angte's to each other tend, to turn each other into a position of parallelism with a force varying inversely as the distance."

The plane in which either element tends to turn is that in which the variation of $\cos \epsilon$ for a given small angle turned through is a maximum. Resolving the elements into their components, we find that the component $i d x$ tends to turn $i^{\prime} d y^{\prime}$ round the axis of $z$, and $i^{\prime} d z^{\prime}$ round the axis of $y$; hence the turning-couple exerted by $i d s$ on $i^{\prime} d s^{\prime}$ is

$$
\mu i i\left\{\left\{\frac{d x}{d s} \frac{d y^{\prime}}{d s^{\prime}}-\frac{d y}{d s} \frac{d x^{\prime}}{d s^{\prime}}\right\} d s d s^{\prime}\right.
$$

round the axis of $z$; and similarly for the other axes.

18. Every elementary current in a given direction, as $i d x$, has at any point in space distant $r$ from it the potential $\frac{i d x}{r}$, which being a vector or directed quantity, namely having the direction $x$, is with propriety termed the vector potential of the element. Maxwell indeed states distinctly (vol. ii. p. 267) 
that the vector potential stands in exactly the same relation to the elementary current in which the potential of a particle of matter stands to that particle-a statement which leads to all the consequences we now point out.

19. Again, the mutual potential energy of any two elementary currents $i d s, i^{\prime} d s^{\prime}$ being $\mu i i^{\prime} \frac{\cos \epsilon}{r^{\prime}} d s d s^{\prime}$, may be put in
the form

$$
\mu\left\{\frac{i \frac{d x}{d s}}{r} i^{\prime} \frac{d x^{\prime}}{d s^{\prime}}+\frac{i \frac{d y}{d s}}{r} i^{\prime} \frac{d y^{\prime}}{d s^{\prime}}+\frac{i \frac{d z}{d s}}{r} i^{\prime} \frac{d \varepsilon^{\prime}}{d s^{\prime}}\right\} d s d s^{\prime} .
$$

Now $i \frac{d x}{d s} d s$ is the component part of the current $i d s$ in the direction of $x$. If we assume, as we must, that the current, instead of flowing in an infinitely thin line, is (like a fluid of finite density) distributed uniformly over a small section perpendicular to $d s$, the $x$ component will in like manner be distributed uniformly over the element of area $d y d z$. If we now write $u, v, w$ for $i \frac{d x}{d s}, i \frac{d y}{d s}, i \frac{d z}{d s}$, when therefore $u, v, w$ are the velocities of the current at $d s$ parallel to the coordinate axes, and in like manner $u^{\prime}, v^{\prime}, w^{\prime}$ for $i^{\prime} \frac{d x^{\prime}}{d s} \& c$, we shall have for the mutual potential energy of the two elements the expression

$$
\mu\left(\frac{u}{r} u^{\prime}+\frac{v}{r} v^{\prime}+\frac{w}{r} w^{\prime}\right) d x d y d z d x^{\prime} d y^{\prime} d z^{\prime}
$$

A similar expression holds for the mutual potential of every pair of elementary currents. We may therefore express the whole potential of any system of currents in the form of a sextuple integral or if

$$
2 \mathrm{~T}=\mu \iiint \iint\left\{\frac{u u^{\prime}+v v^{\prime}+w w^{\prime}}{r}\right\} d x d y d z d x^{\prime} d y^{\prime} d z^{\prime} ;
$$

$$
\begin{gathered}
\iiint_{r} \frac{u}{r} d x^{\prime} d y^{\prime} d z^{\prime}=\mathrm{F}, \\
\iiint_{\int} \frac{v^{\prime}}{r} d x^{\prime} d y^{\prime} d z^{\prime}=\mathrm{G}, \\
\iiint_{2 \mathrm{w}} \frac{w^{\prime}}{r} d x^{\prime} d y^{\prime} d z^{\prime}=\mathrm{H}, \\
\iiint_{2}(\mathrm{~F} u+\mathrm{G} v+\mathrm{H} w) d x d y d z .
\end{gathered}
$$


20. It would appear at first sight that we have omitted the energy of the action of each element on itself, which is prima facie infinite. Exactly in the same way, in finding

$$
\iiint \frac{m m^{\prime}}{\sqrt{x^{2}+y^{2}+z^{2}}} d x d y d z
$$

as the expression for the whole potential energy of a mass of matter, we appear to obtain the energy only of the mutual actions of each pair of particles, and not that of each particle on itself. But if the matter have at every point finite density, it is easily seen that the above expression gives us the whole potential.

In like manner, if the current $u$ or $i d x$ be conceived as flowing, not through an infinitely thin line, but through a tube of finite section, so that the density, so to speak, of the current is finite at every point, the expression

$$
\iint(\mathrm{F} u+\mathrm{G} v+\mathrm{H} w) d s
$$

gives us the whole electrokinetic energy of all the currents in the field. tial.

F, G, H will now be called the components of vector poten-

21. We have hitherto called the above expression

$$
\mu \iiint(\mathrm{F} u+\mathrm{G} v+\mathrm{H} w) d x d y d z
$$

the POTENTIAL energy of the system; and we have seen that it is deduced from our proposed law by methods applicable to potential of mass. We now assume, with Maxwell, that this energy, consisting, as he says it does, of "something in motion and not a mere arrangement," may be treated as kinetic energy for the purpose of applying to it the equation of Lagrange. By this means, as Maxwell has shown, the phenomena of induction are capable of explanation.

22. It is now to be observed that, as above mentioned, and as Maxwell points out, the vector potential of an elementary current in any direction, as $x$, stands in the same relation to the current as the potential of a mass of matter situated at the middle point of the element stands to that matter. It follows that the vector potential has all the properties of the potential of mass. Hence can be most easily deduced many most important theorems in electrodynamics.

For instance $\mathrm{F}$, the vector potential of currents parallel to $x$, must satisfy the equation

$$
4 \pi \mu u+\nabla^{2} \mathrm{~F}=0
$$

(where $u$ is the current in $x$ ), corresponding to Poisson's equation

$$
4 \pi \rho+\nabla^{2} \mathrm{~F}=0
$$


where $\rho$ is density, $\nabla$ potential of mass*. This equation occurs in the electromagnetic theory of light, giving one of the two expressions for $u$, from which arises the equation

$$
\mathrm{K} \mu \frac{d^{2} \mathrm{~F}}{d t^{2}}=\frac{d^{2} \mathrm{~F}}{d x^{2}}
$$

23. Again, it is a known property of the potential of mass, that if the potential of mass $A$ has the same value as that of mass $B$ at every point of a surface completely enclosing both, it has the same value at all points in space outside that surface.

In like manner we conclude that if $\mathrm{F}$, the vector potential of the $x$ component of a system of currents, has the same value as $\mathrm{F}^{\prime}$ (that of another system) at all points of a surface completely enclosing both systems, then $F$ has the same value as $\mathrm{F}^{\prime}$ at all points beyond that surface. Hence can be easily deduced, by way of illustration, the theory of magnetic images as follows.

Let there be an infinite conducting plane, and let a determinate system of electric currents be suddenly generated parallel to and wholly on one side of the plane. Let $u, v$ be the two components of current at any point in the system, $\mathrm{F}, \mathrm{G}$ the components of vector potential of the system. By the principle of least kinetic energy a system of currents will be excited or "induced" in the plane, such as to make the whole energy the least possible consistently with the given currents in the given system. Let $u^{\prime}, v^{\prime}$ be the components of these currents at any point in the plane; $F^{\prime}, G^{\prime}$ the corresponding components of vector potential. Then for the whole energy we have

$$
\begin{aligned}
2 \mathrm{~T} & =\mu \iint\left\{\left(\mathrm{F}+\mathrm{F}^{\prime}\right) u+\left(\mathrm{G}+\mathrm{G}^{\prime}\right) v\right\} d \sigma \text { over the system, } \\
& +\mu \iint\left\{\left(\mathrm{F}+\mathrm{F}^{\prime}\right) u^{\prime}+\left(\mathrm{G}+\mathrm{G}^{\prime}\right) v^{\prime}\right\} d \sigma \text { over the plane. }
\end{aligned}
$$

And in order for $\mathrm{T}$ to be a minimum, given $u$ and $v$ at all points in the system, we must have $\frac{d \mathrm{~T}}{d u^{\prime}}=0, \frac{d \mathrm{~T}}{d v^{\prime}}=0$ at every point in the plane. That is, $F=-F^{\prime}, G=-G^{\prime}$ at every point in the plane; and this being true at every point in the plane, must be true at all points in space beyond the plane.

The system of currents induced in the plane thus at all points beyond the plane exactly neutralizes the given system. It has, in fact, the same effect as the given system reversed in direction.

* The constant $\mu$ depends on the nature of the medium where the currents are situated, and stands in the same relation to the current as $\frac{1}{i}$ ( $w$ here $k$ is the specific inductive capacity of the dielectric) stands to free electricity. 


\section{Messrs. Abney and Festing on the Transmission of}

But the effect of the induced system at points on the same side of the plane with the given system is equal and opposite to its effect at the corresponding points on the opposite side of the plane. Consequently the induced system has at all points in space on the same side of the plane with the given system the same effect as the optical image of the given system formed on the plane as a mirror.

24. We have shown that the proposed law of force leads directly to the correct expression for the energy of any electrical system, whether that energy be termed kinetic or potential, and that by the shortest and easiest method. The further development of the theory would generally take the same form as in Maxwell's 'Electricity,' Maxwell's results being obtained generally by the application of Lagrange's equations to this expression for the energy.

\section{On the Transmission of Radiation of Low Refrangi- bility through Ebonite. By Capt. ABNEY, R.E., F.R.S., and Col. Festevg, R.E.*}

WH HEN Mr. Graham Bell described his interesting experiments with the photophone, we were much surprised to learn that an effect was produced when sheets of ebonite of small thickness were interposed between the apparatus and the source of radiation; and it became a matter of more than curiosity to us to know what was the cause of the phenomenon, since photographic manufacturers were commencing to use ebonite in the construction of the dark slides for the camera.

We think we can demonstrate, however, that the ordinary explanation of transmission of radiant energy can account for the phenomenon. Dr. Guthrie kindly furnished us with a sheet of ebonite, through which the action of a beam of radiation on a selenium cell was most marked; and we accordingly first experimented with that. A photographic spectroscopic apparatus was employed, of the form we have already described in other papers; and the compound of silver was used which is sensitive to all parts of the spectrum. Atfirst we employed only one prism, and used the sun as a source of illumination; and here it may be parenthetically remarked that on the evening when ws made our first experiments the wind was blowing from the north-east, and there was a clear sky. Half the slit was covered up, a piece of ebonite placed in front of the other half, and a plate exposed to the action of the spectrum of the radiations (if any) coming through this thin layer of apparently

* Communicated by the Physical Society, having been read at the Meeting on April 9. 